\title{
Démons et Septante : les raisons d'une enquête
}

Le présent ouvrage se propose d'étudier les représentations des démons et des puissances démoniaques dans la Bible dite des Septante (LXX), en les comparant avec celles qui nous ont été transmises par la Bible hébraïque. Bien que cette recherche fasse amplement recours aux instruments de la philologie et de la critique textuelle, son intérêt ne porte pas sur une analyse strictement philologique, mais vise d'abord et avant tout à mieux cerner le fonctionnement de la catégorie de démon dans les traditions bibliques de langue hébraïque et grecque, et notamment dans le contexte du judaïsme hellénistique, dont la Septante peut être considérée à bien des égards comme un produit fondamental, voire comme le produit fondamental. Un des principes sous-jacents à ce travail est que l'étude des démons dans la Septante a un caractère interculturel intrinsèque, car il implique nécessairement un dialogue avec d'autres représentations du démoniaque produites en langue grecque à la même époque. D'un point de vue plus général, cette étude vise à ouvrir des interrogations nouvelles sur certains aspects de l'imaginaire religieux de l'hellénisme au sens large, ainsi que sur la relation entre les différentes cultures qui font partie de l'hellénisme. À cet égard, l'enquête menée dans les pages qui suivent s'adresse non seulement aux biblistes et aux spécialistes de la Septante, mais également aux historiens qui s'intéressent aux transformations des catégories religieuses dans l'Antiquité, et part du présupposé que la catégorie de démon représente un opérateur culturel important pour étudier ces changements dans les sociétés de la Méditerranée antique ${ }^{1}$. Il me semble, en effet, pouvoir anticiper que les représentations relatives aux démons constituent un bon objet pour une approche «interculturelle» de l'étude de l'Antiquité, non seulement car on retrouve des démons dans toutes les cultures de l'Antiquité méditerranéenne, mais surtout dans la mesure où le savoir relatif aux puissances démoniaques fait l'objet à la fois de continuités et de changements considérables dans différents contextes historiques et religieux. Et c'est notamment dans cette relation complexe entre persistance et changement que les spécificités culturelles les plus significatives se situent, à savoir, dans ce cas, celles qui sont liées à la

1 Voir, à cet égard, les remarques de Petersen 2003. Sur l'imaginaire comme catégorie de l'étude historique, voir les remarques fondamentales de Le Goff 1985, p. v-xxv. 
traduction grecque de la Bible par rapport à sa Vorlage hébraïque, mais aussi au contexte plus large dans lequel cette traduction a été réalisée. Par conséquent, l'étude des démons dans la Septante demande à être approchée par une démarche qui n'est pas seulement interculturelle, mais qui relève véritablement du comparatisme, dans la mesure où la comparaison entre Bible hébraïque et Septante ne peut fonctionner qu'en mettant en perspective les deux contextes respectifs de référence, en amenant ainsi à une confrontation indirecte entre monde sémitique et monde grec.

À cet égard, il importe de relever que les approches normalement utilisées dans l'étude des démons dans la Bible (d'habitude considérée par son texte hébreu) ne manquent pas de soulever certains problèmes méthodologiques, qui pourraient être contournés, sinon résolus, par une approche centrée sur la Septante. Avant de rentrer dans les détails de l'analyse, il convient, donc, d'esquisser brièvement ces problèmes ${ }^{2}$ ainsi que d'expliquer les raisons qui m'ont poussée à mettre la Septante au centre de ma recherche.

Les démons bibliques entre « survivance » et « emprunt »

L'intérêt pour les démons bibliques, à la fois du côté des biblistes et de celui des historiens des religions, remonte au moins au XIX ${ }^{\mathrm{e}}$ siècle ${ }^{3}$ et se poursuit tout au long du siècle dernier ${ }^{4}$, jusqu'à faire l'objet d'études récentes ${ }^{5}$. Malgré des différences parfois importantes du point de vue des outils d'analyse employés et des cadres épistémologiques de référence, on remarque quelques tendances récurrentes à l'égard de la définition et du traitement du démoniaque dans la Bible hébraïque. En particulier, deux paradigmes de recherche me semblent pouvoir être identifiés.

D'une part, on relève l'effort de lister un nombre important de démons, souvent accompagnés par d'autres créatures plus ou moins monstrueuses ou terrifiantes (serpents monstrueux, ombres des morts, géantes, esprits, fantômes,

2 Un parcours historiographique détaillé de l'étude des démons dans la Bible hébraïque se situerait partiellement en dehors du cadre de ce travail. Le paragraphe suivant ne se veut, donc, pas un état exhaustif de la recherche (qui, d'ailleurs, reste encore à écrire), mais un point de synthèse sur les problèmes récurrents posés par certaines tendances dans l'étude des démons bibliques.

3 Voir surtout Baudissin 1876, p. 110-146; De Visser 1880; Wellhausen 1897 ; Robertson Smith, 1889, p. 84-139; Witton Davies 1898.

4 Söderblom 1916; Volz 1924; Langton 1949; Caquot 1956; id. 1971.

5 Voir le volume édité par Lange, Lichtenberger et Römheld 2003; Frey-Anthes 2007; Blair 2009 ; Kitz 2016. 
etc.), ce qui donne lieu à un répertoire dont la nature est en réalité passablement hétérogène ${ }^{6}$. Cet ensemble varié, pourtant classé sous la rubrique unique de «démon », est généralement interprété comme étant une survivance d'une pensée magique et superstitieuse à laquelle l'évolution monothéiste aurait tenté de s'opposer. On a tendance à attribuer à ces survivances des origines diverses, à savoir un emprunt provenant des cultures voisines, notamment babyloniennes ou perses ${ }^{7}$, traditionnellement considérées comme étant spécialisées en «démonologie»; ou alors à postuler un «substrat cananéen » effacé par le Yahvisme, dont la Bible délivrerait encore quelques vestiges ${ }^{8}$. À cet égard, il n'est pas rare de voir les démons relégués au deuxième plan à l'avantage des anges, ces derniers étant regardés comme le seul véritable produit propre à la religion israélite ${ }^{9}$. Cette démarche présente également le risque de projeter en arrière une opposition entre anges et démons qui, comme on le verra, ne se retrouve pas comme telle dans la Bible hébraïque, mais qui est en réalité le reflet d'un développement successif.

D'autrepart, uncourantd'études d'inspirationàlafoisdogmatico-théologique et romantico-phénoménologique, postule l'intégration progressive d'aspects «démoniaques» dans la nature de Yhwh lui-même ${ }^{10}$; l'affirmation de Yhwh comme dieu unique d'Israël ne laisserait alors plus d'espace à la croyance aux démons, au moment où cette divinité assumerait en soi des qualités et des caractères qui, dans d'autres systèmes - supposément dualistes ou polythéistes -, seraient en revanche associés à des puissances multiples. Cela aurait donc amené les Israélites à expérimenter non seulement l'aspect protecteur du dieu, mais également son côté sombre et obscur, effrayant, voire franchement horrible ${ }^{11}$. Il n'est pas difficile de reconnaître dans de telles positions une lecture historicisée de la phénoménologie de Rudolph Otto (notamment de son Das Heilige, 1917), selon lequel l'expérience du sacré comme «ganz anders », altérité irréductible, est par sa nature extraordinaire, à la fois fascinante mais aussi obscure et épouvantable ${ }^{12}$.

6 Voir, à titre d'exemple, Langton 1949, encore régulièrement cité comme ouvrage de référence par les études les plus récentes.

$7 \quad$ Voir encore récemment Riley 1999, p. 235-240.

8 Par exemple Caquot 1956.

9 Caquot 1971; voir encore D.B. Martin 2010.

10 Elle a été théorisée pour la première fois par De Visser dans sa thèse de doctorat (1880), où il soutient notamment que les démons de l'Israël ancien étaient en réalité perçus comme des esprits subordonnés ou intégrés à Yhwh, et ne disposaient pas, par conséquent, d'un pouvoir autonome véritable.

11 Voir à cet égard Söderblom 1916 et Volz 1924.

12 Cette interprétation prévaut encore dans les entrées d'encyclopédies théologiques jusqu'à la fin du XX siècle: Wanke 1981 (TRE); Roehl 1986³ (E KL); Görg $1999\left(\right.$ RGG $\left.^{4}\right)$. 
Ces deux paradigmes de recherche ont traversé le fil des siècles et ont été proposés à plusieurs reprises. Bien que distincts, voire éloignés, l'un de l'autre, ils partagent entre eux le fait d'approcher l'étude de la catégorie du démoniaque dans la perspective du monothéisme en tant que trait distinctif de la religion israélite. Cette perspective devient un point de départ aisé pour la définition des démons comme puissances intrinsèquement négatives - ou, du moins, opposés aux hommes et aux dieux. En outre, elle parvient à expliquer, d'une manière ou d'une autre, la pauvreté de données relatives aux démons dans la Bible hébraïque, qui semble avoir posé souci à la plupart des études sur les démons bibliques ${ }^{13}$. En réalité, comme le note justement Rüdiger Schmitt ${ }^{14}$, la situation israélite ne diffère pas du reste de la documentation levantine de l'âge du Fer; de plus, cette pauvreté de données ne doit pas nécessairement être expliquée par une absence de la croyance dans les démons, mais peut être motivée par d'autres facteurs.

Ces deux démarches paraissent aujourd'hui problématiques, car elles ne semblent pas tenir compte du fait que l'idée de la supposée «révolution monothéiste » ${ }^{15}$ a été sérieusement questionnée par la recherche récente. À cet égard, la vision de la religion israélite comme ayant un caractère exceptionnel par rapport à un prétendu substrat cananéen n'est désormais plus acceptable d'un point de vue historique ${ }^{16}$. En outre, sans vouloir nier l'effective attribution des qualités démoniaques à Yhwh dans plusieurs passages bibliques, il faudrait plutôt comprendre ces aspects du dieu comme faisant partie de la nature nécessairement multiple et complexe qui caractérise toute grande divinité de l'antiquité, laquelle ne peut pas être classifiée tout simplement comme «bienfaisante » ou «malfaisante ».

L'abandon des paradigmes liés au monothéisme comme trait distinctif de la religion de l'Israël ancien, bien que nécessaire, a cependant posé problème aux chercheurs, car il semble avoir ouvert un certain vacuum conceptuel à l'égard à la fois de la définition des démons et de l'étude de leur fonction à l'intérieur des textes bibliques. De cette difficulté ressort l'idée, proposée par la recherche

\footnotetext{
13 Voir encore Van der Toorn 2003.

14 Schmitt 2015. Ce problème fera, notamment, l'objet d'une discussion dans le chapitre suivant.

15 Pour ce concept, voir Assmann 1998.

16 Pour le premier point, voir, entre autres, les essais collectés dans le volume de PongratzLeisten 2011, ainsi qu'Olyan 2012. Pour les continuités entre culture matérielle israélite et celle du reste du Levant, on peut voir, entre autres, Finkelstein et Silberman 2002; Liverani 2003, p. 66 sqq. Isabel Cranz a également souligné la nécessité de sortir de l'opposition entre monothéisme et polythéisme lorsqu'on discute de la présence ou de l'absence des démons dans la Bible hébraïque : voir Cranz 2014.
} 
la plus récente, de renoncer à cette catégorie en ce qui concerne l'étude de la Bible hébraïque, en tant que catégorie non indigène et donc inapte à décrire des phénomènes bibliques ${ }^{17}$.

Nous sommes ainsi passés des modèles qui reconnaissaient une grande quantité «d'emprunts» ou de «survivances » démoniaques, éparpillées à plusieurs endroits de la Bible, à des hypothèses qui éjectent complètement la notion même de «démon » de leur domaine de recherche, en remplaçant cette notion par une série de sous-catégories, qui arrivent parfois à n'inclure qu'un seul membre («puissances du chaos», « divinités mineures », « divinités déchues », « esprits des confins », « génies protecteurs », etc. $\left.{ }^{18}\right)$. Ces démarches semblent donc aboutir à des résultats aporétiques, et soulignent, à mon avis, la nécessité d'un nouvel angle d'approche, qui permette de dépasser les difficultés méthodologiques, notamment celles liées à une interprétation en termes moraux de la nature du démoniaque. À cet égard, Jonathan Z. Smith ${ }^{19}$ signalait déjà la nécessité de sortir d'une définition morale du démoniaque, en la remplaçant par une définition «locative »: il considérait les démons comme des marqueurs taxonomiques, qui n'ont pas, en soi, de traits moraux mais servent à définir une relation (d'opposition ou de distance) par rapport au monde des hommes et à celui des dieux. Une telle approche a le mérite de permettre une construction des démons aussi bien comme figures positives, en tant qu'intermédiaires humano-divins, que négatives, en tant que puissances associées à la dimension du liminaire, du chaos et de l'incontrôlable. Elle touche, en outre, à un aspect central de la notion de démon du point de vue historico-religieux, à savoir la possibilité de définir et délimiter cette notion par rapport à celle, non moins complexe, de «dieu ». D'autres travaux plus récents, comme ceux d'Anders Klostergaard Petersen ${ }^{20}$, ont également avancé des définitions heuristiques ou fonctionnelles de « démon », qui ont été, à l'occasion, exploitées avec succès dans l'analyse de certains dossiers spécifiques dans la Bible hébraïque ${ }^{21}$.

Toutefois, aucun nouveau paradigme général ne semble à présent émerger dans le domaine des études bibliques. Une difficulté supplémentaire à cet égard est représentée par des arguments de nature linguistique, qu'il ne faut pourtant pas surestimer. S'il est vrai que le substantif pour « démon» en français comme dans plusieurs langues européennes remonte étymologiquement au grec, et qu'en revanche le nom de « démon » en hébreu biblique est rare, il

17 Blair 2009, Frey-Anthes 2007.

18 Voir pour une discussion le chapitre 1.

19 J.Z. Smith 1978.

$20 \quad$ Petersen 2003; comparer en outre Twelftree 2007, p. 91 et Barbu et Rendu-Loisel 2009.

21 Voir surtout le travail de Kitz 2016 sur robeș/rabișu, dont je discuterai dans le premier chapitre. 
faut de toute manière éviter le risque de confondre, pour ainsi dire, le mot avec la chose. L'absence d'un mot indigène, ou sa rareté, n'implique pas forcement l'absence de la catégorie sous-jacente. Si tel était le cas, il faudrait d'ailleurs renoncer aux principaux objets détude en histoire des religions antiques (on ne pourrait plus parler, par exemple, du « rite », ni de la « religion » des Grecs ou des Israélites, car ces mots n'apparaissent jamais ni dans la littérature grecque ni dans la Bible hébraïque). À l'inverse, là où le mot est bien attesté, comme dans le cas de $\delta \alpha i \mu \omega \nu$ en grec ancien, les significations associées à ce mot par la culture grecque aux époques archaïque et classique sont très différentes de ce que nous tendons à regrouper aujourd'hui sous la catégorie «démon». Bien plus, il n'est peut-être pas inutile de rappeler que la notion moderne de « démon » ne peut pas être superposée à aucun des concepts des anciens, car elle est le résultat d'une évolution beaucoup plus longue et non linéaire.

Mon propos sera alors celui d'élaborer une définition de travail assez large et inclusive, capable de décrire plusieurs phénomènes dont les acteurs pourraient, à bien des égards, être qualifiés de « démoniaques » dans la perspective des anciens, même si le nom de «démon » n'est pas utilisé par les textes eux-mêmes, à savoir une notion qui permet de garder un équilibre entre le niveau descriptif (emic) et celui redescriptif (etic), ou, en paraphrasant une heureuse formulation de Carlo Ginzburg ${ }^{22}$, entre la possibilité d'avoir accès aux catégories indigènes et la nécessité d'utiliser des instruments conceptuels qui sont, par nécessité, culturellement déterminés.

\section{La place de la LXX dans l'étude de la catégorie du démoniaque}

Il est néanmoins surprenant de constater que, jusqu'à présent, la Septante a occupé une place très limitée dans la discussion sur les démons bibliques.

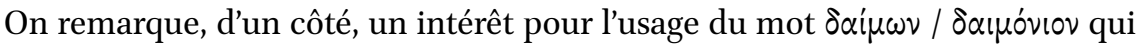
fait l'objet de quelques entrées d'encyclopédie, ou, dans le meilleur des cas, de quelques articles s'interrogeant sur la manière par laquelle l'introduction de ce mot a modifié la représentation des démons dans la Bible ${ }^{23}$. De l'autre côté, un certain nombre d'études techniques ont mis en évidence la complexité des problèmes liés à la traduction en grec de certains passages bibliques qui traitent des démons; elles ont également signalé l'impact du texte de la Septante à

\footnotetext{
22 Ginzburg 2013, p. 201-2O2.

23 Riley 1999, p. 235-240 ; Keel 2003, p. 213-214 ; D.B. Martin 2010, p. 658-666.
} 
l'égard de la perception du démoniaque dans le judaïsme hellénistique : ces travaux restent pourtant limités à des dossiers spécifiques et ponctuels ${ }^{24}$.

De la part des classicistes, la représentation des démons dans la Septante reste largement absente des principales études qui se penchent sur la transformation de la catégorie du «démonique/démoniaque » à l'époque hellénistique, ou sur la naissance d'une démonologie au tournant de l'ère chrétienne ${ }^{25}$.

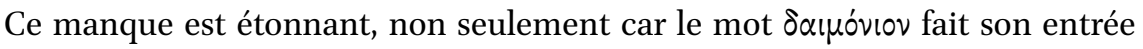
dans les Écritures par le biais de la traduction grecque de la Bible, comme on l'a souvent répété, mais pour toute une série d'autres raisons. L'importance de la Septante comme instrument pour la critique textuelle de la Bible hébraïque, comme Bible des Pères et comme œuvre érudite du judaïsme alexandrin, est bien connue depuis longtemps et ne doit pas être rappelée ici. Mais, au-delà de ces aspects, les dernières années ont témoigné d'un intérêt croissant à l'égard de la Septante qui souligne davantage sa valeur comme objet de synthèse culturelle et, de ce fait, comme produit proprement hellénistique, à la fois du point de vue de son langage ${ }^{26}$ et des représentations culturelles qu'elle véhicule ${ }^{27}$. $\mathrm{Au}$ passage, il vaut la peine de rappeler que d'un point de vue simplement quantitatif la Septante représente le corpus le plus conséquent de littérature postclassique en langue grecque. Au vu de l'importance qui est de plus en plus reconnue à la traduction grecque de la Bible hébraïque, il parait donc pertinent d'essayer de combler ce manque, en élargissant le champ d'études à une analyse complète du domaine du démoniaque dans la Septante, et en croisant ces données avec celles qui ressortent d'autres sources d'époque hellénistique.

Une enquête centrée sur la Septante peut d'ailleurs amener, me semble-t-il, toute une série d'avantages de perspective. Puisque la Septante constitue de facto la première réception de la Bible hébraïque, cette approche permet de récupérer une dimension historique réelle et dynamique dans l'étude des démons, en contournant le risque de se faire prendre au piège des schémas théologiques préconstitués (tels que, notamment, l'opposition prétendue entre polythéisme et monothéisme). L'étude de la catégorie de démon dans ce corpus se justifie d'autant plus par le fait que, en tant que produit culturel hellénistique, la Septante se situe dans une période clé pour la redéfinition de la notion du démoniaque, qui, comme on le verra en détail par la suite, fait l'objet de nouvelles resémantisations à partir de cette époque. Ces changements

24 Voir, entre autres, Schenker 2001 ; Dogniez 2003; Schaper 2010.

25 Voir, par exemple, les travaux de Brenk $(1977,1986$, Brenk et Lanzillotta 2017) ou les études de Sfameni Gasparro (1997, 2009) ; mais également, en ce qui concerne l'Égypte hellénistique, Lucarelli (2011).

26 T. Evans 2001 ; Aitken 2014 b ; J. Lee 2018.

27 Voir, par exemple, Aitken 2007 ; Vergari 2020. 
traversent tant les traditions grecques que le judaïsme ancien, et ils jettent les bases pour le développement d'un véritable savoir démonologique dans l'Antiquité tardive.

Par conséquent, l'adoption d'une perspective centrée sur la traduction grecque de la Bible fournit un point de vue privilégié pour observer des phénomènes de continuité et des processus d'innovation par rapport aux traditions de l'Israël ancien. Par «traditions de l'Israël ancien » j'entends, au sens large, celles qui ont donné origine à la Bible hébraïque mais également celles qui, tout en restant exclues du canon biblique, faisaient intégralement partie du judaïsme antique, telles que les traditions apocryphes et pseudépigraphiques, ou les productions littéraires et liturgiques de Qumrân. En outre, puisque la réalisation de la Septante a nécessairement comporté une négociation avec la culture hellénistique environnante, cette démarche permet d'évaluer les rapports avec les traditions grecques d'époque hellénistique et notamment avec l'hellénisme égyptien, ainsi que les apports de ces traditions à l'imaginaire du démoniaque tel qu'il se construit dans le judaïsme ancien. Réciproquement, l'étude de la Septante dans son contexte hellénistique peut contribuer à son tour à la reconstruction du paysage religieux de cette époque. À cet égard, il est nécessaire de préciser que la notion d'hellénisme à laquelle je fais recours ne se réduit pas à une extension progressive de l'influence grecque sur les autres civilisations du bassin méditerranéen. Au contraire, elle sous-tend un paradigme dynamique et articulé28, caractérisé par une constellation de multiples interactions culturelles, dans lesquelles la Septante participe à plein titre.

En choisissant la Septante comme perspective d'analyse privilégiée, et en écartant les approches traditionnellement employées dans l'étude des démons bibliques que l'on a discutées précédemment, je dois, d'abord, préciser quelle démarche j'entends suivre ici. Cette étude adopte une perspective que l'on pourrait qualifier d'« anthropologie de la traduction », à savoir une méthode d'analyse qui cherche à clarifier la manière dont la traduction était comprise et fonctionnait dans l'Antiquité, du point de vue à la fois des traducteurs et des destinataires anciens de la Septante. Une telle approche permet, à mon avis, de mieux éclairer le rôle joué par la traduction grecque de la Bible dans l'Antiquité, en resituant les représentations véhiculées par la Septante dans leur contexte historique-culturel de provenance, à savoir le scénario intellectuel 
et culturel de l'Égypte hellénisée, du judaïsme hellénistique, voire de l'hellénisme lui-même. Elle a, en outre, l'avantage de respecter le double statut du texte de la Septante, qui doit être compris à la fois comme traduction et comme œuvre autonome ${ }^{29}$. Le potentiel de cette approche a déjà donné des résultats significatifs : il a été notamment mis en évidence par des travaux récents sur l'anthropologie de la traduction dans le monde gréco-romain, ainsi que par les études qui se penchent sur la réception du langage de la Septante dans le christianisme ancien, notamment via les Pères ${ }^{30}$.

Cependant, la nature particulière du corpus constitué par la Septante soulève des problèmes spécifiques, dont il est nécessaire de tenir compte. À l'égard des rapports entre la Bible hébraïque et sa traduction grecque, des difficultés supplémentaires surgissent à cause du fait que nous sommes presque complètement dépourvus de traités ou commentaires qui pourraient nous fournir des indications sur la manière dont la pratique de la traduction était perçue et pratiquée dans ce contexte. Nous n'avons presque rien de comparable aux prologues des comédies de Ménandre ; ni aux traités de Cicéron sur ses traductions du grec ; ni encore aux commentaires par lesquels Jérôme a accompagné sa Vulgate. À cet égard, le peu de documents dont nous disposons, tel que le Prologue du Siracide ou la lettre d'Aristée, laissent plutôt entendre que la traduction de la Bible hébraïque a été réalisée en suivant une logique bien différente de celle selon laquelle fonctionnaient normalement les traductions dans le monde classique : ces témoignages montrent, en effet, un souci de fidélité et de littéralisme qui était étranger, par exemple, aux auteurs romains lorsqu'ils traduisaient du grec ${ }^{31}$. En revanche, l'analyse de la langue de la Septante et la comparaison avec d'autres documents de l'Égypte ptolémaïque suggèrent qu'il vaut mieux comprendre le travail des traducteurs à la lumière des traditions scribales qui opèrent dans un contexte marqué par un bilinguisme important, voire dans un contexte franchement multilingue. Ce contexte parait également caractérisé par une activité continue à la fois de traduction et de production littéraire, qui deviennent, à un moment donné, indissociables ${ }^{32}$.

29 Sur le double statut de la Lxx, voir Muraoka 2001.

30 Sur le premier aspect voir, par exemple, Passoni Dell'Acqua 2008; Bettini 2012 ; sur le deuxième Bons, Brucker et Joosten 2014.

31 Voir sur ce point B. Wright 2003; Bettini 2012. La situation est compliquée ultérieurement lorsque l'on tient compte du fait que les considérations du neveu de Ben Sira et de l'auteur anonyme de la Lettre d'Aristée sont inspirées par un souci de légitimation de la LXx. Par conséquent, il n'est pas assuré que leur insistance sur la nature extrêmement littérale de la traduction ait effectivement représenté un souci majeur pour les premiers traducteurs grecs.

Voir Aitken 2014 b ; id. 2016. 
Il faut ensuite considérer les problèmes posés par l'absence de données concernant non seulement les rédacteurs du texte-source, qui demeurent anonymes mais également les traducteurs du texte-cible, dont on ne sait presque rien ; et, encore, les circonstances historiques effectives dans lesquelles la traduction grecque de la Bible s'est déroulée, qui nous échappent ${ }^{33}$.

Enfin, on ne peut pas oublier que la Vorlage de la Septante constitue un obstacle en soi. Le fait que la Septante ne traduit pas le texte massorétique tel que nous l'avons aujourd'hui, mais un texte qui pouvait être à bien des égards différent ${ }^{34}$, et - ce qui est plus problématique - un texte que dans la plupart des cas nous n'arrivons plus à reconstruire avec précision, rend l'analyse des rapports entre la Septante et la Bible hébraïque une affaire encore plus délicate.

Compte tenu de ces difficultés, il paraît d'autant plus nécessaire de compléter une approche qui se veut anthropologique par d'autres outils méthodologiques, tels qu'ils nous sont fournis par la philologie et la critique textuelle, la lexicographie et l'analyse sémantique, ainsi que par les translation studies, et notamment par l'analyse des techniques de traductions, dont les potentialités méthodologiques et les résultats concrets dans le domaine de la Septante ont été désormais mis en évidence ${ }^{35}$.

Méthodologie

Les remarques ci-dessus m’ont amenée à développer une approche intégrée qui combine (a) philologie et critique textuelle; (b) analyse lexicale et sémantique ; (c) analyse des styles de traduction. L'enquête philologique et textuelle (a) est une prémisse incontournable en vue de l'établissement du texte et de l'évaluation des choix de traduction. À cet égard, une attention particulière est dédiée aux passages dans lesquels la Septante atteste d'un original hébreu différent du texte massorétique et aux variantes qui sont appuyées par la comparaison avec d'autres témoins, tels que les fragments de Qumrân. Dans la mesure du possible, j'ai également pris en compte les processus de transmission du texte grec en analysant les variantes attestées par les réviseurs (Aquila, Symmaque et Théodotion) ainsi que par les recensions (surtout en ce qui concerne le texte

33 La bibliographie sur ce sujet est, désormais, devenue très vaste, et sur les circonstances d'origine de la Septante nous sommes encore loin d'une position partagée parmi les chercheurs. L'étude de Honigman 2003 reste un bon point de référence pour une synthèse du débat.

34 Voir, à ce sujet, par exemple, Schorch 2006 ; De Troyer 2013.

35 Voir, entre autres, les volumes édités par Peters 2006; id. 2013, ainsi que les études de Brock 1979; Aejmelaeus 2007; van der Louw 2006, 2007, 2013. 
dit lucianique). L'intégration de ces textes dans le travail est essentielle car le refus ou l'acceptation de certains choix de traduction fournissent des éléments utiles en vue d'évaluer la relation des communautés juives avec leur milieu environnant; du point de vue de l'étude de la tradition manuscrite, ces éléments peuvent donner des indications précieuses sur la manière dont le texte grec a été corrigé sur la base d'un texte hébreu plus tardif, fournissant ainsi des informations sur les développements internes à la Septante elle-même. L'analyse philologique a souvent été complétée par une analyse lexicale et sémantique (b) à la fois de l'hébreu et du grec. L'analyse des domaines sémantiques du vocabulaire du «démoniaque » ainsi que de ses usages métaphoriques et métonymiques est particulièrement propice pour mettre en miroir les représentations culturelles véhiculées respectivement par le texte hébreu et par le texte grec. Elle s'avère ainsi être un outil fondamental pour identifier des trajectoires d'innovation et/ou de continuité. Enfin, si l'analyse du dossier grec et hébreu qui traite du démoniaque ne peut pas faire abstraction de l'étude des techniques de traductions employées pour chaque passage (c), il importe toutefois de préciser que ma démarche ne vise pas à étudier ces techniques comme un objet de recherche en soi, mais plutôt en tant qu'outil méthodologique pour une meilleure compréhension du fonctionnement de la traduction dans son ensemble ${ }^{36}$. À cet égard, en suivant les remarques d'Anneli Aejmelaeus ${ }^{37}$, le cœur de mon analyse vise à mettre en évidence les dynamiques à l'œuvre dans la traduction de la Septante entre la décodification du langage du texte-source, c'est-à-dire l'hébreu, et sa re-codification dans la langue-cible, le grec. J'ai, donc, commencé par prendre en compte les critères sémantiques et discursifs qui peuvent à chaque fois motiver certains choix de traduction; les différents niveaux d'interprétation du texte qu'une traduction peut véhiculer par rapport à une autre; ainsi que le rapport avec la traduction du Pentateuque comme possible source lexicographique faisant autorité38. Ce n'est qu'une fois les autres hypothèses exclues que l'hypothèse d'une exégèse, voire d'une réinterprétation, a été envisagée, tout en tenant compte du fait qu'une partie du corpus est constituée par des textes traditionnellement considérés comme des «traductions libres », c'est-à-dire des traductions dans lesquelles on enregistre le plus grand nombre de déviations par rapport à l'hébreu. En adoptant cette perspective, j'essaie, dans la mesure du possible, de

36 Je souscris, en ce sens, aux affirmations de Lemmelijn 2001.

37 Aejmelaeus $1991 ; 2007$.

38 Dogniez 2001 ; Pietersma 2006 a ; sur l'influence exercée par la traduction du Pentateuque sur les autres livres, voir l'étude classique de Tov 1981 et récemment Olofsson 2013, pour une position plus nuancée. 
dépasser l'opposition traditionnelle entre traduction littérale et traduction libre qui, bien que partiellement connue des anciens, était perçue de manière différente dans l'Antiquité par rapport à aujourd'hui. Les recherches de James Barr et d'autres critiques ont, par ailleurs, depuis longtemps montré que le littéralisme n'est pas tant une stratégie délibérée des traducteurs anciens que la technique de traduction la plus simple à utiliser ${ }^{39}$.

Ces considérations m'amènent également à exercer une certaine prudence à l'égard d'autres approches méthodologiques qui, dans un sens ou dans l'autre, ne font pas droit à ce que Takamitsu Muraoka a défini comme «la double nature de la $\mathrm{LXX} »^{40}$. Il s'agit, d'un côté, de l'approche narratologique, laquelle considère les traducteurs comme des narrateurs et interprète les déviations par rapport au texte de référence comme étant en lien avec un projet global de traduction, projet dont l'existence est pourtant indémontrable ${ }^{41}$. De l'autre côté, le paradigme interlinéaire me paraît également problématique. D’après ce paradigme, la langue de la Septante témoignerait d'une dépendance étroite à l'hébreu qui ne la rendrait jamais complètement autonome. La dépendance du grec à l'égard de l'hébreu procèderait vraisemblablement d'une dépendance fonctionnelle (la traduction ayant servi comme base pour l'étude de la Bible hébraïque dans des contextes d'école $)^{42}$. Malgré la pertinence de plusieurs observations de nature linguistique, la théorie sur les origines et sur la fonction première de la traduction qui est impliquée par ce modèle demeure spéculative, et elle n'est pas complètement satisfaisante. Le dossier des démons me semble au contraire démontrer que, si l'on considère la Septante dans son ensemble comme une œuvre de longue durée, et si l'on prend en compte ses développements internes, ce texte présente des caractères indéniables d'autonomie, et ce, en dépit des intentions qui pourraient avoir été à l'origine de la traduction en grec du Pentateuque. En outre, l'assomption de l'«interférence » de l'hébreu sur le grec comme principe d'analyse qui est à la base de ce paradigme ne me paraît pas toujours vérifiée par une analyse détaillée du texte grec. De plus, un tel principe s'avère problématique dans la mesure où il implique une séparation rigide entre traduction et production littéraire, deux processus qui font l'objet d'une articulation plus complexe dans l'activité scribale de l'époque, et qu'il vaut mieux aborder par une approche intégrée.

\footnotetext{
39 Barr 1961. Sur la nécessité de dépasser l'opposition entre traduction littérale et libre voir récemment Dhont 2019.

40 Muraoka 2001.

41 Beck 2000, voir, à ce sujet, les remarques de van der Louw 2013.

42 Pour une formulation récente et nuancée de ce paradigme voir Pietersma 2010.
} 
La démarche comparative qui caractérise cette approche définit en même temps le noyau géographique et chronologique du dossier qui sera analysé dans les pages qui suivent. Ce dossier est, en principe, constitué par les textes de la Septante et par ceux de la Bible hébraïque. Sous la rubrique «Septante», je comprends les écrits traduits et composés en grec environ entre le $\mathrm{III}^{\mathrm{e}}$ et le $\mathrm{I}^{\mathrm{er}}$ siècle av. n. è., en laissant de côté les questions liées à la canonisation de ladite «Bible grecque» dans l'Antiquité. Le dossier cherche également à tenir compte de la pluralité interne de la tradition manuscrite de la Septante, en examinant les variantes textuelles disponibles en grec; en ce qui concerne l'hébreu, la complexité de la relation entre le texte grec et sa Vorlage oblige à prendre en compte les témoins extérieurs au texte massorétique (le Pentateuque samaritain, les témoignages de Qumrân et la Vieille Latine lorsqu'ils ont été préservés). En revanche, d'autres écrits juifs hellénistiques tel que les œuvres de Philon et de Josèphe, ainsi que certains textes de la littérature du Second Temple, comme les livres d'Hénoch et des Jubilés, seront abordés seulement de manière ponctuelle et non systématique. Bien que ces œuvres nous transmettent des informations significatives sur la démonologie de l'époque, elles posent une série de problèmes spécifiques qui méritent à leur tour une analyse séparée. Elles ont d'ailleurs fait l'objet de plusieurs travaux récents, dont les résultats seront pris en compte et mis en perspective avec les données provenant de la Septante.

Les représentations du démoniaque dans la Bible hébraïque et dans la Septante sont d'abord encadrées par leurs contextes matériels et culturels de référence : l'Israël ancien et le Levant, d'un côté (chapitre 1); la culture grecque et plus particulièrement hellénistique de l'autre (chapitre 2). La représentation des démons dans le Levant est inscrite dans un horizon de comparaison plus large qui fait appel à la Mésopotamie et l'Égypte, alors que le traitement des démons en Grèce aborde, entre autres, le thème de la polysémie du $\delta \alpha i \mu \omega \nu$ et

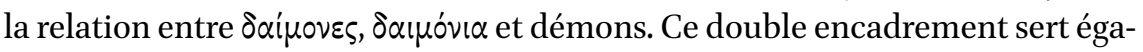
lement à établir une définition du démoniaque qui soit opérationnelle dans les deux contextes. Par ce biais, on arrive à discuter la notion de $\delta \alpha i \mu \omega v / \delta \alpha \mu o^{\prime} v i o v$ à l'époque hellénistique ainsi que sa signification dans la Septante (chapitre 3). À cet égard, il faut toutefois préciser que mon but n'est ni d'établir un champ sémantique univoque du démoniaque, car il s'agirait d'une opération impossible ou du moins fictive, ni de retracer une théologie de la Septante comme un système uniforme et cohérent ${ }^{43}$. Je vise plutôt à reconstruire un imaginaire 
religieux et culturel dont la nature est nécessairement multiple et plurielle, selon les différents contextes dans lesquels la notion de démon intervient ainsi que selon les différences spécifiques qui caractérisent chacune des traductions et des compositions de la Septante. À ce propos, au lieu d'étudier chaque substantif ou chaque nom hypothétique de démon dans sa singularité, il m’a paru plus pertinent de repérer une série de scénarios ou domaines principaux, dans lesquels la représentation des démons est thématisée dans le texte grec ou dans le texte hébreu. L'identification de ces domaines n'a pas été établie en choisissant a priori le texte grec ou le texte hébreu comme point de départ, mais relève déjà d'une comparaison entre les deux : les différents scénarios ont été déterminés par la présence du mot $\delta \alpha i \mu \omega \nu / \delta \alpha \mu \delta^{\prime} v ı v$ ainsi que par d'autres critères de nature linguistique ou thématique.

Avant de procéder à l'identification des domaines du démoniaque, je discute néanmoins certains cas dans lesquels des figures de démon de premier rang de la Bible hébraïque semblent avoir complètement disparu, ou avoir fait l'objet d'une transformation considérable dans la traduction grecque (chapitre 3). Le dossier le plus significatif à cet égard est celui d'Azazel, énigmatique destinataire du bouc émissaire envoyé dans le désert, au cours du rituel d'expiation pour la purification du temple dans le chapitre 16 du Lévitique. Les questions relatives à son nom et sa fonction, liées à l'originalité du rituel qui le concerne, ont fait l'objet d'un débat qui a parfois remis en question son identité démoniaque. En dépit d'interprétations contraires, la construction syntaxique des passages de Lev 16 mentionnant Azazel ne permettent pas, à mon sens, de le comprendre autrement que comme une entité surnaturelle présidant des espaces liminaires tels que, notamment, le désert (Lev 16,10b) $)^{44}$. La question de la réception d'Azazel dans la Septante du Lévitique reste relativement peu étudiée; ce phénomène est en réalité plus complexe qu'on ne l'a dit parfois, comme le montrent notamment les différents choix de traduction repérables dans les passages où il est nommé, ou encore les divergences entre la Septante et les autres versions anciennes. Je m'interroge en particulier sur la signification du prétendu néologisme $\delta \dot{\alpha} \pi \circ \pi \circ \mu \pi \alpha \hat{\imath} \circ \varsigma$, « l'éliminateur », au verset 8 et 10a, ainsi que sur les raisons qui ont poussé le traducteur à ne pas conserver ce choix de traduction dans la suite du chapitre (aux versets 1ob et 26). Un cas de figure comparable à celui d'Azazel est celui de Lilith, dont la nature démoniaque a parfois été remise en question dans la Bible hébraïque mais qui, à la lumière des parallèles levantins et mésopotamiens, ainsi que d'autres

44 Sur la nature d'Azazel comme un démon dans la Bible hébraïque, voir également Cranz 2014, p. 84-86. 
traditions du Second Temple, ne peut pas être niée. Comme dans le cas d'Azazel, Lilith fait l'objet d'une re-sémantisation particulière dans la Septante qui transforme, sans pourtant l'oblitérer complètement, son caractère de démon. En revanche, la Septante ne préserve aucune trace d'une prétendue conception démoniaque du péché, qui dans le texte hébreu du récit de Caïn et Abel est tapi à la porte et guette Caïn $($ Gen 4,7$)$ : à cet égard il faut toutefois observer que nous avons affaire à un passage dont le sens exact demeure assez obscur déjà dans le texte de départ.

La comparaison entre texte grec et hébreu fait ressortir quatre contextes principaux où le motif des démons est prééminent. Le premier est celui des agents divins envoyés par Yhwh et responsables des maladies et des fléaux, tels que Resheph, Deber, Qețeb, le « destructeur » qui tue les premiers nés des Égyptiens, les anges de malheur, et d'autres figures associées à la manifestation de la colère de Yhwh (chapitre 4). Certaines de ces puissances, comme Qețeb et Deber, disparaissent complètement dans la Septante (par exemple en Deut 32,24), bien qu'il reste à déterminer si ce phénomène correspond à une volonté délibérée d'oblitération de ces figures ou, simplement, à une perte de référent chez les traducteurs grecs. D'autres, comme Resheph, sont réinterprétés selon des paradigmes qui mettent en avant des aspects animaliers, liés notamment aux oiseaux rapaces, et qui, dans certains cas, semblent refléter un arrière-plan égyptianisant: ce sera notamment le cas de la réception de Resheph en Hab 3,5 dans la version dite Barberini, qui fait l'objet d'une analyse spécifique. Dans d'autres cas, l'imaginaire mis en place par la Bible hébraïque est remplacé par un nouvel imaginaire qui augmente considérablement la nature démoniaque de ces puissances. C'est notamment le cas du Psaume 91 (= 9o LXX) où, dans le grec, les noms de Deber et Qețeb s'évanouissent pour

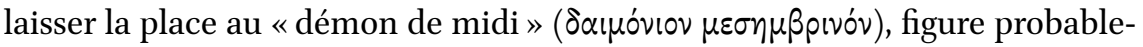
ment née d'une lecture erronée du texte mais qui jouera un rôle important dans la démonologie des Pères de l'Eglise. Cette figure semble inspirée d'un ensemble de traditions grecques, bien attestées à l'époque hellénistique, relatives au milieu de la journée comme moment redoutable car ouvrant le passage aux apparitions surnaturelles, et pouvant présenter un danger pour les humains. J'examinerai en outre la question de savoir dans quelle mesure l'introduction de ce démon dans le Ps 91 doit éventuellement être rapprochée de la tradition qumrânienne qui associe étroitement ce texte avec des pratiques d'exorcisme (11QPsAp ${ }^{\mathrm{a}}$ [11 11$\left.]\right)$. Dans ce contexte, un dernier agent qui semble acquérir une importance considérable dans le texte grec est la parole de Yhwh (Logos). La représentation du Logos en tant qu'hypostase de Yhwh associée à son activité créatrice est bien connue par la Bible hébraïque; en revanche, 
ses qualités «démoniaques» sont davantage accentuées par les traducteurs grecs et semblent faire l'objet d'un développement interne aux traditions de la Septante.

Un deuxième contexte d'action du «démoniaque » est repérable dans une série de passages prophétiques en Isaïe et Jérémie, ainsi que dans d'autres textes prophétiques, qui font des démons les principaux habitants du paysage des ruines (chapitre 5). Les passages qui décrivent la destruction de villes et le repeuplement de ruines par des animaux sauvages et des démons sont caractérisés par une série d'isotopies, telles que l'hétérogénéité, la pollution et le chaos, et le motif semble particulièrement lié à la destruction de Babylone. L'analyse porte davantage sur les chapitres 13 et 34 du livre d'Isaïe qui représentent le cœur de ce corpus (c'est également dans cette liste que Lilith fait son unique apparition dans la Bible hébraïque, en Is 34,14). Si nous considérons le grec, nous nous trouvons, désormais, face à un bestiaire distinct, à savoir des sirènes, des onocentaures, des hérissons et des démons $(\delta \alpha \mu o ́ v i \alpha)$. L'hypothèse de travail est que le traducteur grec a cherché à peupler les lieux désertiques d'Is 13 et 34 avec des figures de la marginalité qui lui étaient connues et accessibles, et qu'il a interprété le potentiel démoniaque présent dans le texte hébreu à la lumière des nouveaux paradigmes épistémiques de l'époque hellénistique associant animaux exotiques et entités démoniaques.

Un troisième contexte qui voit les démons à l'œuvre dans la Septante est celui de la polémique contre les dieux des autres (chapitre 6). Les termes $\delta \alpha i \mu \omega \nu / \delta \alpha \mu o_{v}{ }^{\circ}$ v traduisent en hébreu šẽdîm, 'élilîm et Gad; ce phénomène demande par conséquent un examen spécifique. Les šêdîm sont identifiés à des nouveaux dieux qui font l'objet d'un culte étranger (Deut 32,17; Ps 106,37); alors que 'êlil, littéralement «vain, inconsistant», désigne souvent les idoles (Ps 96,5). On a donc ici, d'une part, la première attestation d'un déplacement sémantique qui revient à « démoniser» les dieux des autres; d'autre part, les indices d'un rapprochement entre la notion de démon et celle d'idole. De ce point de vue, une piste de recherche importante, et qui n'a pas été explorée jusque-là, consiste à considérer différents passages de Plutarque qui semblent témoigner d'une certaine fluidité entre idoles ( $\varepsilon^{\prime \prime} \delta \omega \lambda \alpha$ ) et démons ( $\left.\delta \alpha \iota \mu o ́ v i \alpha\right)$, et qui discutent la question de la possibilité de sacrifier aux démons. Le rapport entre Gad et $\delta \alpha i \mu \omega \nu$ est par contre analysé dans une autre perspective, en par-

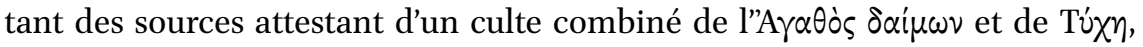
personnification de la Fortune, dans l'Égypte hellénistique.

Le rapport entre démons et esprits constitue une autre piste féconde de recherche, qui fait l'objet d'un traitement spécifique au chapitre 7. Le terme rûah ou $\pi v \varepsilon v \hat{\mu} \alpha$ n'ayant, en soi, de connotation ni positive ni négative, il est employé pour définir un attribut direct de la divinité, et il est particulièrement 
associé aux phénomènes de possession prophétique. Il peut également parfois désigner une puissance terrifiante. Mon intérêt porte en particulier sur l'analyse des épisodes où des esprits, sans nom propre mais souvent qualifiés par des adjectifs, agissent sur les hommes en manifestant une tendance de plus en plus autonome : je compare à cet égard le récit du premier livre des Rois ( $1 \mathrm{R} 22$ ), où un esprit se propose lui-même à la cour de Yhwh pour servir comme « esprit de mensonge » en vue de séduire le roi d'Israël, avec le rôle des esprits dans l'histoire de Saül et David dans le premier livre de Samuel (1 S 16-18). Parmi les réactions mises en ouvre pour combattre l'action des esprits, j'examine notamment la construction de la figure de David comme «exorciste», qui délivre Saül de son esprit par une thérapie musicale, ainsi que le développement de ce motif dans les traditions de la Septante, qui semblent réinterpréter la maladie de Saül à la lumière des catégories de la médecine grecque. Après avoir analysé les recoupements entre les domaines sémantiques de rûaḥ hébreu et de $\pi v \varepsilon \hat{\mu} \mu \alpha$ grec, je discute enfin la possibilité que d'autres passages de la Bible hébraïque et de la Septante fassent référence à des esprits dotés d'une certaine autonomie de Yhwh, notamment dans le cas de l'« esprit d'impureté » mentionné en Zach 13,2; je discute encore le rôle joué par la traduction grecque à l'égard du développement d'une véritable pneumatologie.

À cet égard, une nouveauté importante attestée par la Septante est le rapprochement entre anges et esprits, tel qu'il émerge dans certains passages des Psaumes, comme par exemple le Psaume 104, et dans l'épithète «Seigneur des esprits ». Le rapport entre anges, esprits et démons et l'articulation de leurs rôles à l'intérieur de la cour céleste constitue donc le dernier terrain d'enquête de cet ouvrage (chapitre 8). Je me penche notamment sur les transformations des catégories du mal'āk «messager» et du śạtāan «adversaire», lorsqu'ils indiquent des fonctionnaires de la cour céleste. Dans la Bible hébraïque mal'âk désigne un médiateur céleste qui joue souvent un rôle herméneutique par rapport aux annonces délivrées par les dieux aux hommes (et parfois des hommes aux dieux), et qui souvent surveille l'accomplissement des ordres divins. Il ne correspond donc pas à un « ange » comme être ailé et merveilleux faisant partie d'une troupe hiérarchiquement organisée et opposée à celle des démons, bien que certains passages des Psaumes semblent comprendre déjà les mal'ākîm comme une classe d'êtres surnaturels. De même, le śățān, utilisé quatre fois pour des êtres divins (Num 22, Job 1-2; Zach 3; 1 Ch 21-22), n'est pas dans la Bible hébraïque le nom propre de l'ennemi juré de Yhwh, mais correspond plutôt à la fonction de «l'adversaire » - voire, dans un contexte juridique, de l'«accusateur» plus spécifiquement. Pour trois de ces occurrences,

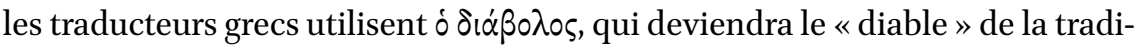
tion apocryphe, apocalyptique et chrétienne. J'essaie alors d'évaluer si, et dans 
quelle mesure, ce terme exprime encore la fonction d'un adversaire de Yhwh en conformité avec le texte hébreu, ce que semble suggérer un ensemble d'indices tels que la présence de l'article en grec ou encore l'emploi dans un passage d'une forme verbale ( $\delta เ \alpha \beta \dot{\alpha} \lambda \lambda \varepsilon v \nu$, «s'opposer»), et dans quelle mesure il indique déjà dans le nom d'une entité autonome et maléfique, comme il paraît évident dans un passage du livre de la Sagesse $(2,24)$. En analysant les développements d'un savoir démonologique et angélologique dans la Septante, je discute également la question de savoir jusqu'à quel point les tendances repérables dans la Septante prolongent ou sont à mettre en parallèle avec des tendances similaires repérables dans des traditions tardives de la Bible hébraïque telles qu'elles apparaissent, par exemple, dans le livre des Chroniques.

Dans ce cadre, j'analyse enfin l'opposition entre l'ange Raphaël et le démon Asmodée dans le livre de Tobit. En tant qu'exemple de roman hellénistique de diaspora, Tobit est un témoin significatif des transformations de l'imaginaire démoniaque du judaïsme d'époque hellénistique, dans la mesure où il propose une représentation de démons comme des forces autonomes et maléfiques, qui ne semblent plus être reliées de manière organique aux systèmes traditionnels de représentation du monde divin. Lange Raphaël, à son tour, fait désormais partie d'une hiérarchie angélique bien organisée et son rôle dans le récit atteste d'un développement considérable des fonctions attribuées aux anges. Je cherche également à déterminer dans quelle mesure la représentation des démons dans le livre est à mettre en rapport avec le genre littéraire de ce recueil ainsi qu'avec son milieu de production et de première réception, en considérant la possibilité que certains détails du récit, tels que l'emploi d'un foie de poisson pour chasser le démon, renvoient à des techniques d'exorcisme effectivement pratiquées dans certains milieux juifs de l'époque du Second Temple. Je m'intéresse enfin à certaines variantes manuscrites qui expliquent l'action du démon par le fait que ce dernier était amoureux de Sara, ainsi qu'à la signification de ces variantes pour l'évolution de l'imaginaire du démoniaque dans les communautés juives hellénistiques.

Enfin, les conclusions présentent une synthèse des principaux résultats du travail, et réfléchissent à ses implications plus larges pour l'étude de la notion de démon dans l'Antiquité. Les enjeux de cette étude portent notamment sur les appellations des démons et le rapport entre leurs dénominations et leurs fonctions; sur le rôle des démons par rapport aux formes traditionnelles de la représentation des puissances divines dans l'Ancien Testament telles que la cour céleste; sur l'encadrement du savoir démonologique, angélologique et pneumatologique dans le contexte du développement progressif d'une vision dualiste du monde; sur les rapports entre possession prophétique et possession démoniaque; sur les origines des traditions exorcistiques dans 
l'Antiquité classique et proche-orientale, ainsi que sur les possibles trajectoires des influences réciproques. J'attire enfin l'attention sur des enjeux d'ordre méthodologique plus général, liés à une prise en compte sérieuse de la Septante dans les études bibliques à la fois pour l'étude la religion de l'Israël ancien et pour l'appréciation de la pluralité interne du judaïsme antique. À cet égard, les résultats de mon étude soulignent la nécessité de repenser les modèles herméneutiques qui décrivent les rapports entre production et transmission du texte biblique, et montrent le potentiel de la Septante pour un renouvellement de tels modèles. Ces résultats montrent enfin la contribution non négligeable amenée par la Septante à l'étude des phénomènes religieux de l'époque hellénistique, phénomènes pour lesquelles ce corpus s'avère comme l'un des témoins les plus anciens et les plus conséquents. 